\title{
Gastric bronchogenic cysts: A case report and literature review
}

\author{
CHAOYONG TU ${ }^{1}$, JINGDE ZHU ${ }^{1}$, CHUXIAO SHAO $^{1}$, WEIBO MAO $^{2}$, XINGMU ZHOU $^{2}$, \\ QIAOMEI LIN ${ }^{1}$, ZHUKAI LI ${ }^{1}$, JIE ZHANG ${ }^{1}$, QINGYUE ZHOU ${ }^{1}$ and WEI CHEN ${ }^{3}$ \\ Departments of ${ }^{1}$ General Surgery and ${ }^{2}$ Pathology, Lishui Central Hospital, Lishui, Zhejiang 323000; \\ ${ }^{3}$ Department of Hepatobiliary and Pancreatic Surgery, Second Affiliated Hospital, \\ Zhejiang University School of Medicine, Hangzhou, Zhejiang 310009, P.R. China
}

Received November 19, 2014; Accepted January 29, 2016

DOI: $10.3892 /$ etm.2016.3067

\begin{abstract}
Gastric bronchogenic cysts are rare lesions, first described in 1956, with only 34 cases reported in the literature to date. The present study described a case of bronchogenic cyst of the stomach in a 17-year-old female who presented with periodic epigastric pain. In addition, the study analyzed the existing literature on these lesions. Gastric bronchogenic cysts are more common in females (female:male ratio, 21:14) and the median age of their development is 43 years. In total, $48.57 \%$ of the 34 previously reported cases were identified incidentally, and the remainder presented mainly with epigastric pain. Cyst sizes varied between 1.7 and $15 \mathrm{~cm}$. In 3 cases, preoperative diagnosis was performed using needle biopsy, whereas several studies were initially misdiagnosed as stromal tumors. In $85 \%$ of the cases $(31 / 35)$, cyst resection was performed, with laparoscopy used in 4 of the cases. The findings of the present study and literature review suggested that bronchogenic cysts of the stomach are rare, and surgical resection is warranted to treat symptoms and prevent malignant transformation.
\end{abstract}

\section{Introduction}

Bronchogenic cysts are rare congenital cystic lesions that are induced by the abnormal budding of the primitive tracheobronchial tube. The location of bronchogenic cysts is dependent upon the embryonic stage of development at which abnormal budding occurs $(1,2)$. The majority of these cysts are located in the mediastinum, whereas the remainder of cases predominantly involve the lung parenchyma $(1,3)$. Abdominal bronchogenic cysts are very rare, particularly those located in the gastric wall (4). Dewing et al (5) were the first to describe such a case in 1956. Gastric bronchogenic cysts are threefold more common in women, in contrast with retroperitoneal

Correspondence to: Dr Wei Chen, Department of Hepatobiliary and Pancreatic Surgery, Second Affiliated Hospital, Zhejiang University School of Medicine, 88 Jiefang Road, Hangzhou, Zhejiang 310009, P.R. China

E-mail: viogro@163.com

Key words: bronchogenic cyst, stomach cystic masses or cutaneous bronchogenic cysts, which appear with equal frequency in men and women (6). Most patients in previous reports were asymptomatic (7-9), but epigastric pain, nausea and vomiting are the most common symptoms that present. Clinical manifestations of gastric bronchogenic cysts are usually associated with cyst enlargement, secondary infection, perforation or compression of adjacent tissues (10). Most gastric bronchogenic cysts are diagnosed postoperatively (11-13), but a number have been carefully monitored without resection (14). In the present study, a case of abdominal bronchogenic cyst in a 17-year-old patient was presented, and the existing literature (Table I) was reviewed in order to analyze the clinical features of these cysts.

\section{Case report}

A 17-year-old Chinese female was admitted to Lishui Central Hospital (Lishui, China) in October 2013 suffering from nonradiating periodic epigastric pain, without a fever. The pain had begun 2 years previously and had increased in severity within the 2 months prior to admission. The patient did not experience nausea, vomiting, dysphagia, heartburn, weight loss, diarrhea, constipation or early satiety, and had no premedical or family history of gastric cancer. All procedures conducted in the present study were approved by the ethics committee of Lishui Central Hospital (Lishui, China), and written informed consent was obtained from the patient and her father prior to participation in the current study. Upon physical examination, no symptoms of note were detected. Blood and stool routine examination, $\alpha$-fetal protein concentration, carcinoembryonic antigen and carbohydrate antigen CA 199 levels were all normal.

A gastroscopic examination revealed a large and mobile submucosal lesion in the cardia, with a diameter of $3 \mathrm{~cm}$ (Fig. 1). The mucosa was smooth and the cardiac ostium of the stomach was mildly strictured, with no evidence of bleeding or ulceration. Pinch biopsies demonstrated a normal gastric mucosa without Helicobacter pylori infection; therefore, gastric submucosal leiomyomatosis was suspected. Endoscopic ultrasonography (EUS) detected a large submucosal lesion in the cardia and the mucosa was smooth. A medium and hypoechoic mass measuring $3 \mathrm{~cm}$ in diameter was detected in the muscularis propria, which was subsequently diagnosed as a stromal tumor (Fig. 2). Using abdominal multi-detector-row 

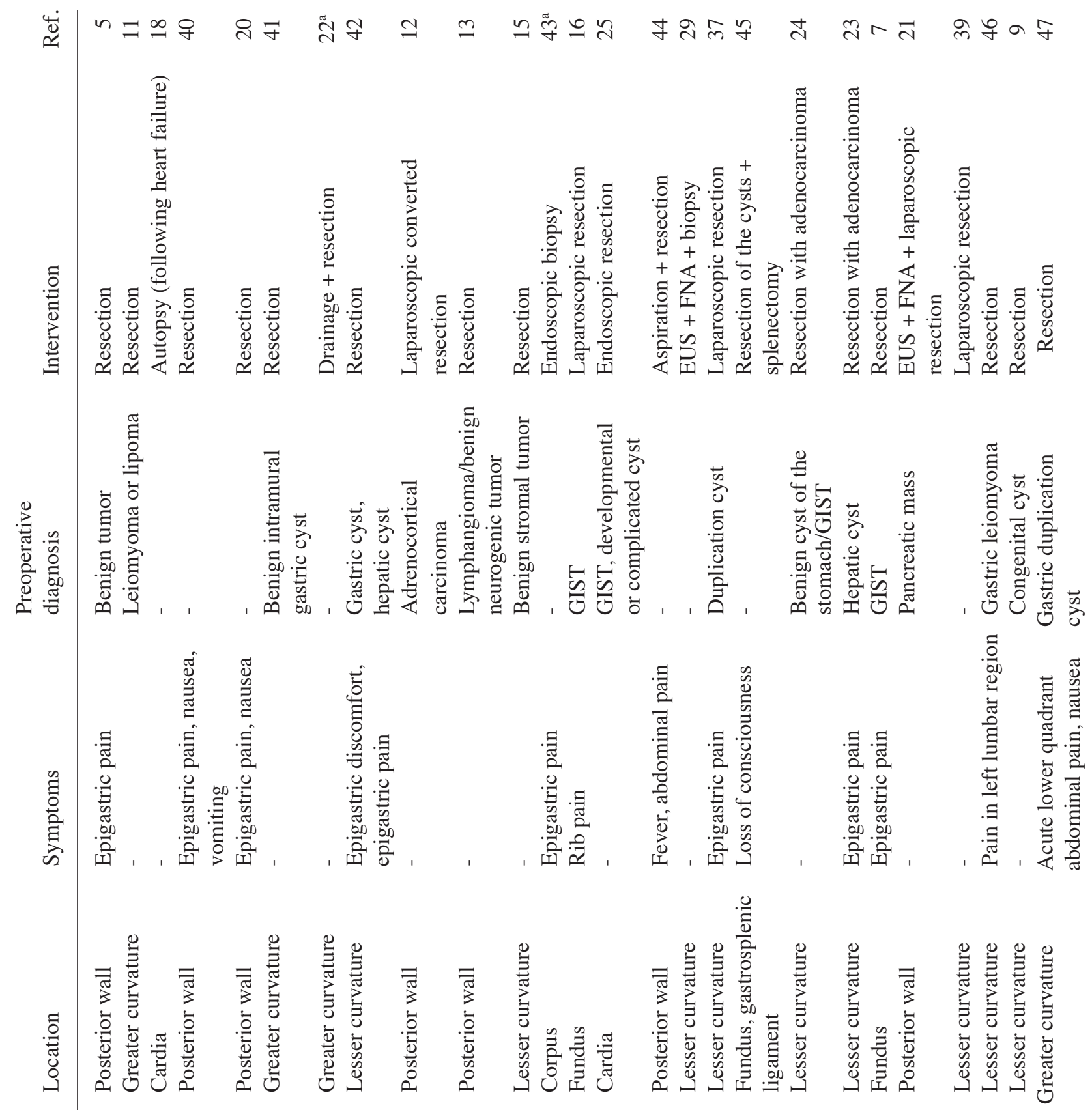

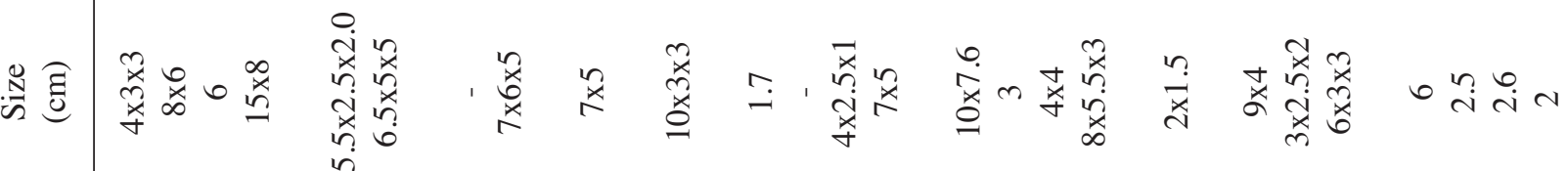

$\frac{\overrightarrow{8}}{\overrightarrow{0}}$

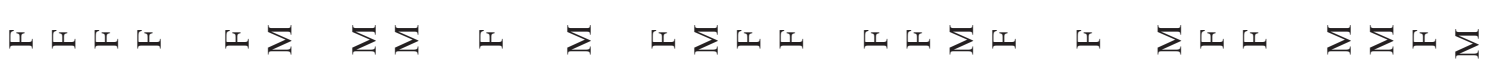

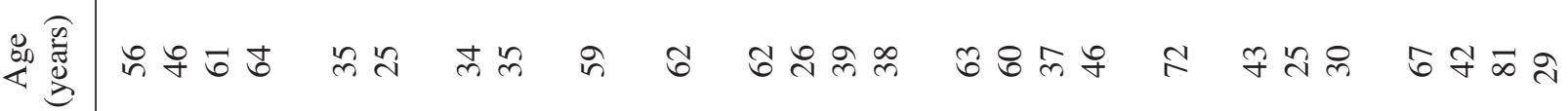




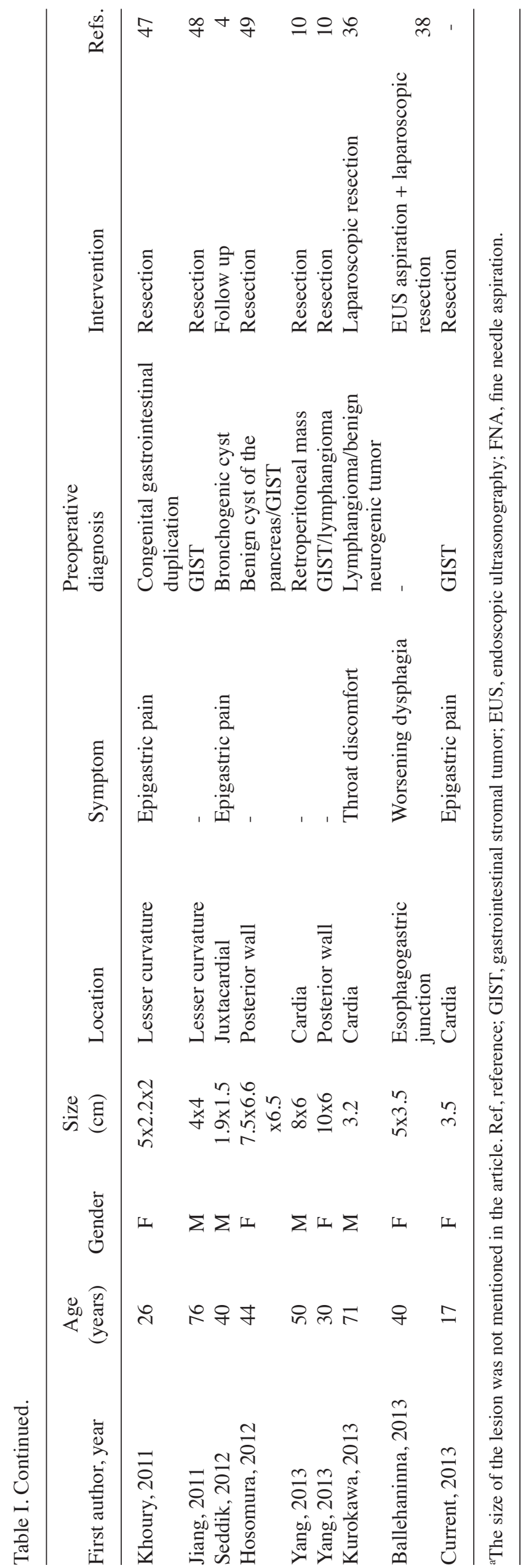

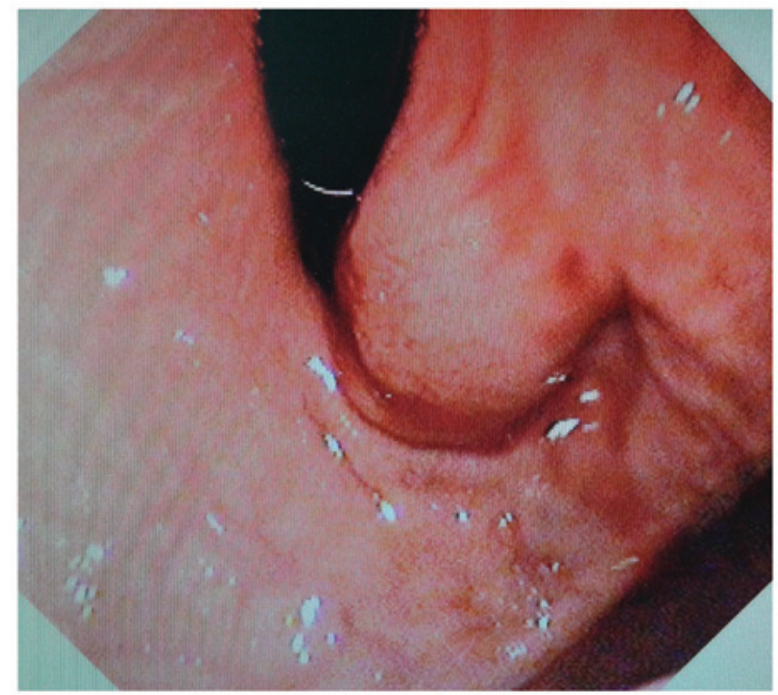

Figure 1. Gastroscopy demonstrated a submucosal lesion.

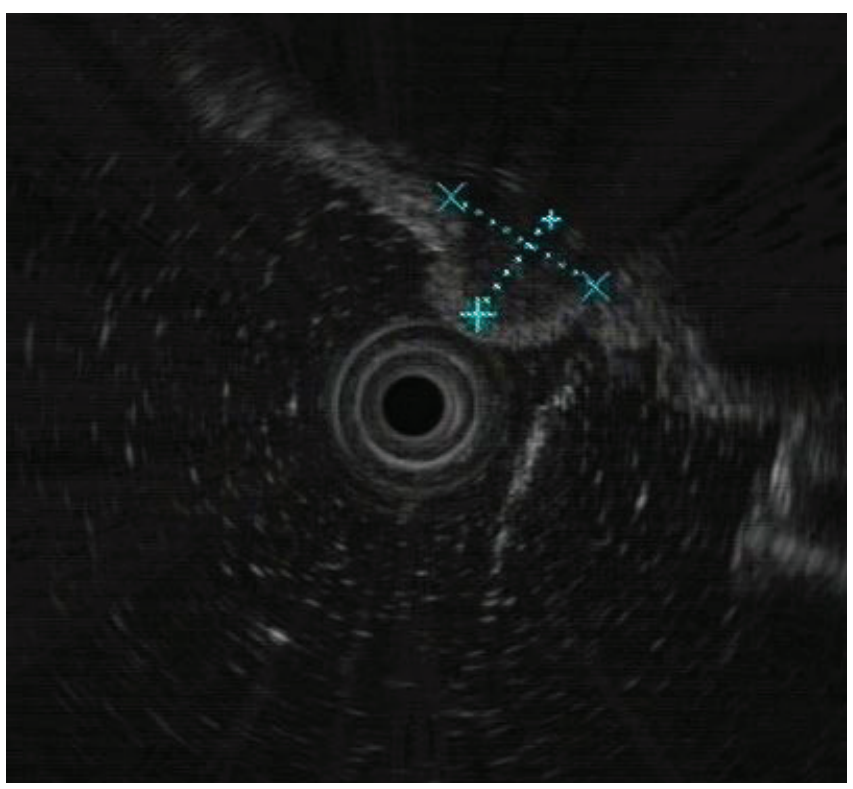

Figure 2. Endoscopic ultrasonography confirmed a medium and hypoechoic mass in the muscularis propria in the cardia.

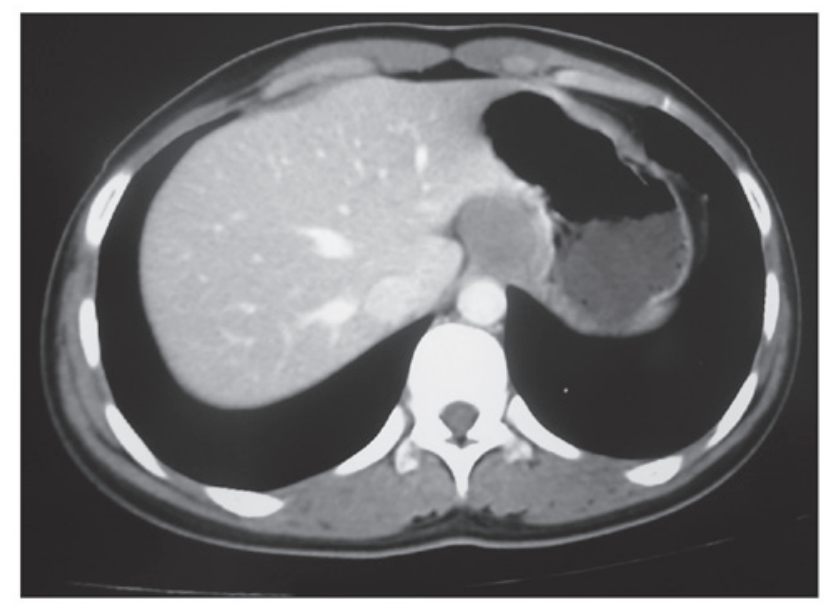

Figure 3. Contrast computed tomography of the upper abdomen demonstrated a non-enhanced lesion in the cardia of the stomach. 


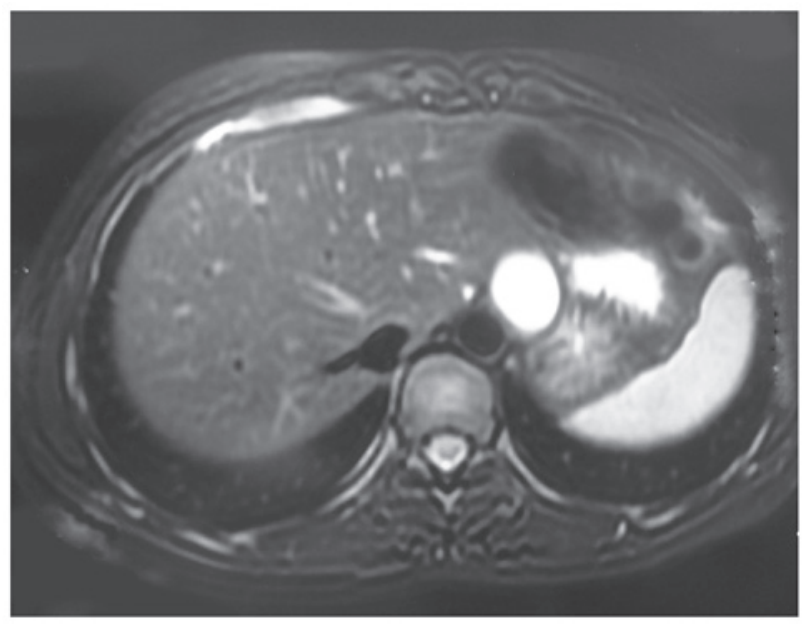

Figure 4. Magnetic resonance imaging of the upper abdomen revealed a high T2 signal focus.

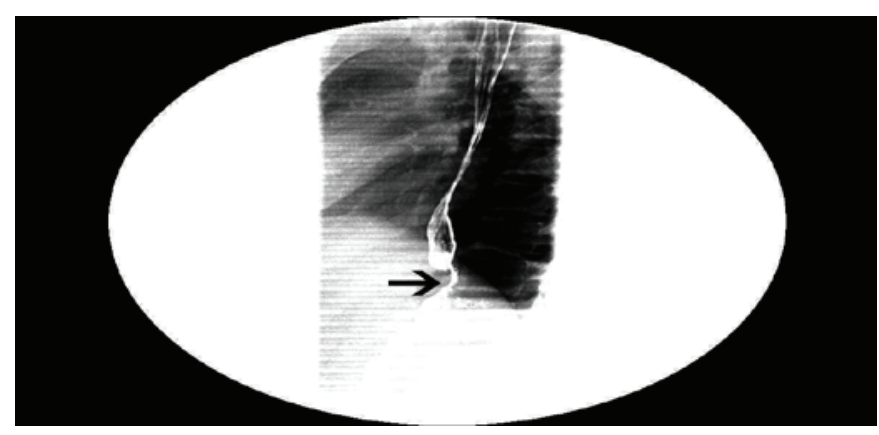

Figure 5. Esophagus barium opacification revealed that the cardia of stomach was compressed. Arrow indicates area of compression.

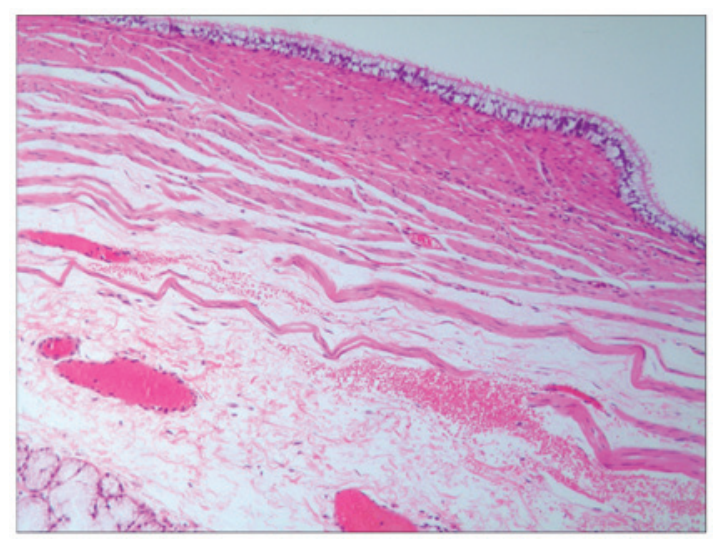

Figure 6. Histological overview using hematoxylin and eosin staining demonstrated that the cystic wall was lined with ciliated respiratory epithelia without cytologic atypia. Original magnification, x100.

computed tomography (CT), a quasi-circular lesion of cystic density without enhancement was detected in the cardia that measured $3.0 \times 2.5 \mathrm{~cm}$. No calcification or septation was detected (Fig. 3). Subsequent magnetic resonance imaging (MRI) demonstrated a smooth and quasi-circular lesion with high T2 and equal T1 signal intensity without enhancement; therefore, a cyst in the cardia of the stomach was suspected (Fig. 4). Esophagus barium opacification revealed that the cardia of the stomach was compressed (Fig. 5). Based on these results, the patient was preoperatively diagnosed with a stromal tumor in the cardia of the stomach.

During an exploratory laparotomy, a smooth cystic mass was detected between the submucosa and seromuscular layers, which measured $3.0 \times 2.5 \mathrm{~cm}$. The lumen of the stomach was intact and no discrete nodules were noted. The mass was successfully resected. Subsequent inspection of the specimen demonstrated that it was cystic and filled with viscous, mucoid cream-colored fluid. Microscopic analysis of the sections was conducted with a Microm HM 325 rotary microtome (Thermo Fisher Scientific, Waltham, MA, USA) following hematoxylin and eosin staining (Sinopharm Chemical Reagent Co., Ltd., Shanghai, China). This revealed that the cystic wall of the cardia consisted of smooth muscle fibers with focal mucous glands and ciliated columnar epithelia without any cytologic atypia. Thus, the diagnosis of a bronchogenic cyst in the cardia of the stomach was confirmed based on its histological appearance (Fig. 6), in accordance with a previous report (15). Postoperatively, the patient recovered successfully and the epigastric pain disappeared. At the 10 month follow-up, a total abdominal CT revealed no recurrence of lesions. At a 12 month follow-up, there was no evidence of recurrence and, as of the last follow-up in December 2014, the patient had reported no symptoms such as nausea, epigastric pain or abdominal distention.

\section{Discussion}

In order to more extensively study bronchogenic cysts of the stomach, these were researched using the keywords 'bronchogenic cyst of the stomach' in Medline/PubMed (www.ncbi.nlm.nih.gov/pubmed). The final date of data collection was in August 2014. All cases confirmed by pathology, and cases with imaging characteristics typical of bronchogenic cysts of the stomach were included, regardless of patient age and gender. Cases without typical pathological or imaging features were excluded, and this search only included papers published in English. To the best of our knowledge, as of August 2014, 34 cases of gastric bronchogenic cysts had been reported under these search conditions. These cases are listed in Table I.

Bronchogenic cysts result from a developmental malformation in the foregut during weeks 3-7 of embryonic development. Migration of these cysts can occur when the attachments to the trachea or esophagus are not maintained (16). Bronchogenic cysts are lined with cuboida or pseudostratified ciliated epithelium and may or may not be surrounded by elastic fibres, smooth muscle and cartilage. The predominant locations include the hilar and middle mediastinal area, whereas extrathoracic and subdiaphragmatic bronchogenic cysts are rare (15). There are $<100$ subdiaphragmatic bronchogenic cyst cases reported in the literature (17), but cases of cysts in the gastric wall are rare. Gastric bronchogenic cysts exhibit a higher prevalence in females (female to male ratio, 21:14), with a median age of development at 43 years old. Only 1 previous case was reported to be multifocular. Of the cases reported to date, $48.57 \%$ (17/35) were asymptomatic and arbitrarily located $(16,18)$; however, a number of patients with gastric bronchogenic cysts have presented with abdominal pain, with 
or without accompanying diseases, such as peptic ulcers or cholelithiasis (15).

Gastric bronchogenic cysts are most commonly located posterior to or on the posterolateral side of the stomach near the gastroesophageal junction. The size of cyst can vary between $1.7 \mathrm{~cm}$ and $15.0 \mathrm{~cm}$ in diameter. Notably, $80 \%$ of gastric duplication cysts do not locate to, nor interact with the mucosa (19), and only 2 cases of intramucosal cysts have been reported $(20,21)$. In the remainder of the identified cases of gastric bronchogenic cysts, the cyst was continuous with the stomach wall or surrounded by smooth muscle that is continuous with the muscle of the stomach. With the exception of elevated CA 19-9 levels in 2 of the cases $(8,22)$, other tumor markers in the majority of cases were all normal, suggesting that there are no specific tumor markers for this condition. Postoperatively, the elevated CA 19-9 levels were found to be normalized. Certain cases also presented with gastric carcinoma $(23,24)$; chronic inflammation of the gastric mucosa resulting from the bronchogenic cyst may have caused adenocarcinoma in the stomach (23). The present study demonstrated that gastroscopy and imaging examinations may be able to locate the lesion; however, they cannot provide qualitative diagnoses. However, Lee et al (25) suggested that when a lesion is suspected to be a solid tumor on the basis of EUS and CT investigations and has a positive pillow sign (a surface indentation observed upon pushing the mass with closed biopsy forceps), the possibility of a bronchogenic cyst should be investigated. Several of these lesions are misdiagnosed as solid mass lesions based on CT and MRI results (26), which may be attributed to the thick proteinaceous contents of these cysts (27).

Radiographically, bronchogenic cysts are typically cystic in nature and compress adjacent structures (28). It is difficult to distinguish them from other cysts solely on the basis of imaging. Ubukata et al (9) demonstrated that far greater clarity was achieved when using MRI, as compared with CT, and emphasized the applicability of this method. In the present case, the high signal intensity on T1-weighted and T2-weighted images suggested that the mass contained mucinous or proteinaceous fluid. EUS is not useful for demonstrating the submucosal location of the cyst, but it may help to distinguish masses that involve the posterior stomach from masses associated with the tail of the pancreas or the left adrenal gland. It has previously been demonstrated that, when clinically indicated, EUS-fine needle aspiration (FNA) biopsy can be used in the definitive diagnosis of gastric bronchogenic cysts (2), particularly as EUS-FNA has a sensitivity of $93-95 \%$, a diagnostic rate of $82-86 \%$, and a low complication rate of only 1-3\% (29-31). Such complications include recurrence, ulceration, infection and hemorrhage. Clinicians should be vigilant for these complications as they may further complicate surgical intervention due to the associated adhesion (32). In the 35 cases analyzed in the present study, 3 cases were preoperatively diagnosed by needle biopsy, whereas the majority of the remaining cases were misdiagnosed as stromal tumors or other benign lesions and one previous case was misdiagnosed due to concomitant gastric carcinoma (23).

When a cyst is enlarged and becomes symptomatic, surgery can be complicated and hazardous. Furthermore, it has previously been demonstrated that gastric bronchogenic cysts are capable of malignant transformation (33-35). Therefore, resection is recommended once the lesion has been identified. However, Seddik et al (4) reported a patient with an unchanged lesion at a 3-year follow-up. Sato et al (29) demonstrated another case which was diagnosed by endosonography-guided FNA biopsy without surgical resection. The results of the present literature review suggest that small and asymptomatic cases should be carefully followed up; however, for symptomatic cases, such as the one described in the present case report, resection is recommended. In the present case, the epigastric pain resulted from the expanding mass pressing on the cardia, which was misdiagnosed as a stromal tumor due to its rarity. In order to decrease postoperative complications and enhance recovery, laparoscopic or endoscopic surgeries remain the first choice for treatment (36-39). Care should be taken in order to avoid intraoperative rupture of the cyst, particularly with infected cysts where postoperative infectious complications are common. Since the gastric mucosa is easily injured and anastomosis performed under laparoscopy may result in postoperative anastomosis leakage, open surgery was selected in the present case, as the gastric bronchogenic cyst was located in the submucosa of the cardia.

In conclusion, gastric bronchogenic cysts are rare and the symptoms are atypical. CT, MRI and EUS-FNA may be selectively used for evaluation; however, the recommendation of surgical intervention for asymptomatic cases remains controversial.

\section{References}

1. Berrocal T, Madrid C, Novo S, Gutiérrez J, Arjonilla A and Gómez-León N: Congenital anomalies of the tracheobronchial tree, lung and mediastinum: Embryology, radiology, and pathology. Radiograhis 24: e17, 2004.

2. Ko SF, Hsieh MJ, Lin JW, Huang CC, Li CC, Cheung YC and $\mathrm{Ng} \mathrm{SH}$ : Bronchogenic cyst of the esophagus: Clinical and imaging features of seven casese. Clin Imaging 30: 309-314, 2006.

3. Aktoğu S, Yuncu G, Halilcolar H, Ermete S and Buduneli T: Bronchogenic cysts: Clinicopathological presentation and treatment. Eur Respir J 9: 2017-2021, 1996.

4. Seddik H, Adyoui T, Rouibaa F, El Hamdi FZ, Aourarh A Mahi M, Benkirane A and Zentar A: Gastric bronchogenic cyst presenting as a submucosal mass: A case report. J Med Case Rep 6: 262-264, 2012.

5. Dewing SB, Roessel CW and Olmstead EV: Enterogenous cyst of the stomach wall, a rare benign lesion; case report. Ann Surg 143: 131-135, 1956.

6. Haddadin WJ, Reid R and Jindal RM: A retroperitoneal bronchogenic cyst: A rare cause of a mass in the adrenal region. J Clin Pathol 54: 801-802, 2001.

7. Jiang L, Jiang L, Cheng $\mathrm{N}$ and Yan L: Bronchogenic cyst of the gastric fundus in a young woman. Dig Liver Dis 42: 826, 2010.

8. Tan KK, Nandini CL and Ho CK: A case of gastric bronchogenic cyst in Singapore with multiple intrigues. ANZ J Surg 80: 286-287, 2010.

9. Ubukata H, Satani T, Motohashi G, Konishi S, Goto Y, Watanabe Y, Nakada I and Tabuchi T: Intra-abdominal bronchogenic cyst with gastric attachment: Report of a case. Surg Today 41: 1095-1100, 2011.

10. Yang $\mathrm{X}$ and Guo K: Bronchogenic cyst of stomach: Two cases report and review of the English literature. Wien Klin Wochenschr 125: 283-287, 2013.

11. Gensler S, Seidenberg B, Rifkin H and Rubinstein BM: Ciliated lined intramucosal cyst of the stomach: Case report and suggested embryogenesis. Ann Surg 163: 954-956, 1966.

12. Hedayati N, Cai DX and McHenry CR: Subdiaphragmatic bronchogenic cyst masquerading as an 'adrenal incidentaloma'. J Gastrointest Surg 7: 802-804, 2003. 
13. Matsubayashi J, Ishida T, Ozawa T, Aoki T, Koyanagi Y and Mukai K: Subphrenic bronchopulmonary foregut malformation with pulmonary sequestration-like features. Pathol Int 53: 313-316, 2003

14. Seddik H, Adioui T, Rouibaa F, El Hamdi FZ, Aourarhz A, Mahi M, Benkirane A and Zentar A: Gastric bronchogenic cyst presenting as a submucosal mass: A case report. J Med Case Rep 6: 262-264, 2012

15. Song SY, Noh JH, Lee SJ and Son HJ: Bronchogenic cyst of the stomach masquerading as benign stromal tumor. Pathol Int 55 : 87-91, 2005.

16. Melo N, Pitman MB and Rattner DW: Bronchogenic cyst of the gastric fundus presenting as a gastrointestinal stromal tumor. J Laparaendosc Adv Surg Tech A 15: 163-165, 2005.

17. Liang MK, Yee HT, Song JW and Marks JL: Subdiaphragmatic bronchogenic cysts: A comprehensive review of the literature. Am Surg 71: 1034-1041, 2005.

18. Shireman PK: Intramural cyst of the stomach. Hum Pathol 18: 857-858, 1987.

19. Spivak H, Pascal RR, Wood WC and Hunter JG: Enteric duplication presenting as cystic tumors of the pancreas. Surgery 121 597-600, 1997.

20. Laraja RD, Rothenberg RE, Chapman J, Imran-ul-Haq and Sabatini MT: Foregut duplication cyst: A report of a case. Am Surg 61: 840-841, 1995.

21. Rubio CA, Orrego A and Willén R: Congenital bronchogenic cyst in the gastric mucosa. J Clin Pathol 58: 335, 2005.

22. Ikehata A and Sakuma T: Gastric duplication cyst with markedly elevated concentration of carbohydrate antigen 19-9. Am J Gastroenterol 95: 842-843, 2000.

23. Shibahara H, Arai T, Yokoi S and Hayakawa S: Bronchogenic cyst of the stomach involved with gastric adenocarcinoma. Clin J Gastroenterol 2: 80-84, 2009.

24. Murakami S, Isozaki H, Shou T, Sakai K and Toyota H: Foregut duplication cyst of the stomach with pseudostratified columnar ciliated epithelium. Pathol Int 58: 187-190, 2008.

25. Lee SH, Park DH, Park JH, Kim HS, Park SH, Kim SJ and Oh MH: Endoscopic Mucosal resection of a gastric bronchogenic cyst that was mimicking a solid tumor. Endoscopy 38 (Suppl 2): E12-E13, 2006.

26. Fazel A, Moezardalan K, Varadarajulu S, Draganov P and Eloubeidi MA: The utility and the safety of EUS-guided FNA in the evaluation of duplication cysts. Gastrointest Endosc 62: 575-580, 2005

27. Wiech T, Walch A and Werner M: Histopathological classification of nonneoplastic and neoplastic gastrointestinal submucosal lesions. Endoscopy 37: 630-634, 2005.

28. Sashiyama H, Miyazaki S, Okazaki Y, Kaiho T, Nakajima Y, Hoshino T, Akai T, Nabeya Y, Funami Y, Shimada H, et al: Esophageal bronchogenic cyst successfully excised by endoscopic mucosal resection. Gastrointest Endosc 56: 141-145, 2002.

29. Sato M,Irisawa A, Bhutani MS, Schnadig V, Takagi T, Shibukawa G, Wakatsuki T, Imamura H, Takahashi Y, Sato A, et al: Gastric bronchogenic cyst ciagnosed by endosonographically guided fine needle aspiration biopsy. J Clin Ultrasound 36: 237-239, 2008.

30. Matsui M, Goto H, Niwa Y, Arisawa T, Hirooka Y and Hayakawa T: Preliminary results of fine needle aspiration biopsy histology in upper gastrointestinal submucosal tumors. Endoscopy 30: 750-755, 1998.

31. O'Toole D, Palazzo L, Arotçarena R, Dancour A, Aubert A, Hammel P, Amaris J and Ruszniewski P: Assessment of complications of EUS-guided fine-needle aspiration. Gastrointest Endosc 53: 470-474, 2001.
32. Vos CG, Hartemink KJ, Golding RP, Oosterhuis JW and Paul MA: Bronchogenic cysts in adults: Frequently mistaken for a solid mass on computed tomography. Wien Klin Wochenschr 123: 179-182, 2011.

33. Endo $\mathrm{C}$, Imai T, Nakagawa $\mathrm{H}$, Ebina $\mathrm{A}$ and Kaimori $\mathrm{M}$ : Bronchioloalveolar carcinoma arising in a bronchogenic cyst. Ann Thorac Surg 69: 933-935, 2000

34. Sullivan SM, Okada S, Kudo M and Ebihara Y: A retroperitoneal bronchogenic cyst with malignant change. Pathol Int 49: 338-341, 1999.

35. Kuraoka K, Nakayama H, Kagawa T, Ichikawa T and Yasui W: Adenocarcinoma arising from a gastric duplication cyst with invasion to the stomach: A case report with literature review. J Clin Pathol 57: 428-431, 2004.

36. Kurokawa T, Yamamoto $M$, Ueda $T$, Enomoto $T$, Inoue $K$, Uchida A, Kikuchi K and Ohkohchi N: Gastric bronchogenic cyst histologically diagnosed after laparoscopic excision: Report of a case. Int Surg 98: 455-460, 2013.

37. Wakabayashi H, Okano K, Yamamoto N, Suzuki Y, Inoue H, Kadota K and Haba R: Laparoscopically resected foregut duplication cyst (bronchogenic) of the stomach. Dig Dis Sci 52: 1767-1770, 2007.

38. Ballehaninna UK, Shaw JP and Brichkov I: Subdiaphragmatic bronchogenic cyst at the gastroesophageal junction presenting with Dysphagia: A case report. Surg Laparosc Endosc Percutan Tech 23: e170-e172, 2013.

39. Díaz Nieto R, Naranjo Torres A, Gómez Alvarez M, Ruiz Rabelo JF, Pérez Manrique MC, Ciria Bru R, Valverde Martínez A, Roldán de la Rúa J, Alonso Gómez J and Rufián Peña S: Intra abdominal bronchogenic cyst. J Gastrointest Surg 14: 756-758, 2010.

40. Braffman B,Keller R, Gendal ES and Finkel SI: Subdiaphragmatic bronchogenic cyst with gastric communication. Gastrointest Radiol 13: 309-311, 1988

41. Takahara T, Torigoe T, Haga H, Yosida I, Takeshima S, Sano S, Ishii Y, Furuya T, Nakamura E and Ishikawa M: Gastric duplication cyst: Evaluation by endoscopic ultrasonography and magnetic resonance imaging. J Gastroenterol 31: 420-424, 1996.

42. Kim JH, Kim JS, Nam ES and Shin HS: Foregut duplication cyst of stomach. Pathol Int 50: 142-145, 2000.

43. Rubio CA, Orrego A and Willen R: Bronchogenic gastric cyst. A case report. In Vivo 19: 383-385, 2005.

44. Cunningham SC, Hansel DE, Fishman EK and Cameron JL: Foregut duplication cyst of the stomach. J Gastrointest Surg 10: 620-621, 2006

45. Theodosopoulos T, Marinis A, Karapanos K, Vassilikostas G, Dafnios N, Samanides L and Carvounis E: Foregut duplication cysts of the stomach with respiratory epithelium. World J Gastroentero 113: 1279-1281, 2007.

46. Mardi K, Kaushal V and Gupta S: Foregut duplication cysts of stomach masquerading as leiomyoma. Indian J Pathol Microbiol 53: 160-161, 2010.

47. Khoury T and Rivera L: Foregut duplication cysts: A report of two cases with emphasis on embryogenesis. World J Gastroenterol 17: 130-134, 2011.

48. Jiang W, Zhang B, Fu YB, Wang JW, Gao SL, Zhang SZ and Wu YL: Gastric duplication cyst lined by pseudostratified columnar ciliated epithelium: A case report and literature review. J Zhejiang Univ Sci B 12: 28-31, 2011.

49. Hosomura N, Kono H, Kawaida H, Amemiya H, Itakura J and Fujii H: A case of foregut gastric duplication cyst with pseudostratified columnar ciliated epithelium. Clin J Gastroenterol 5: 82-87, 2012. 